\title{
Current status and potential challenges of mesenchymal stem cell-based therapy for malignant gliomas
}

\author{
Qing Zhang ${ }^{1 \dagger}$, Wei Xiang ${ }^{1 \dagger}$, Dong-ye $\mathrm{Yi}^{1}$, Bing-zhou Xue ${ }^{1}$, Wan-wan Wen², Ahmed Abdelmaksoud ${ }^{1}$,
} Nan-xiang Xiong ${ }^{1}$, Xiao-bing Jiang ${ }^{1}$, Hong-yang Zhao $^{1}$ and Peng Fu ${ }^{{ }^{*}}$ (D)

\begin{abstract}
Glioma, which accounts for more than $30 \%$ of primary central nervous system tumours, is characterised by symptoms such as headaches, epilepsy, and blurred vision. Glioblastoma multiforme is the most aggressive, malignant, and lethal brain tumour in adults. Even with progressive combination treatment with surgery, radiotherapy, and chemotherapy, the prognosis for glioma patients is still extremely poor. Compared with the poor outcome and slowly developing technologies for surgery and radiotherapy, the application of targeted chemotherapy with a new mechanism has become a research focus in this field.

Moreover, targeted therapy is promising for most solid tumours. The tumour-tropic ability of stem cells, including neural stem cells and mesenchymal stem cells, provides an alternative therapeutic approach. Thus, mesenchymal stem cell-based therapy is based on a tumour-selective capacity and has been thought to be an effective anti-tumour option over the past decades. An increasing number of basic studies on mesenchymal stem cell-based therapy for gliomas has yielded complex outcomes.

In this review, we summarise the biological characteristics of human mesenchymal stem cells, and the current status and potential challenges of mesenchymal stem cell-based therapy in patients with malignant gliomas.
\end{abstract}

Keywords: Mesenchymal stem cells (MSCs), Gliomas, MSC-based therapy, Current status, Potential challenges

\section{Background}

Gliomas are the most common aggressive primary central nervous system tumours in adults [1], and they lead to a severe economic burden all over the world $[2,3]$. Currently, the standard therapy for malignant gliomas is maximal safe surgical resection, postoperative radiotherapy, and then concomitant and adjuvant chemotherapy with temozolomide [4]. Due to the invasive growth features of malignant gliomas, complete resection is rarely achieved. Intra-operative fluorescence-guided technology, such as 5-aminolevulinic acid (5-ALA), could increase the resection proportion but does not improve the overall survival of patients with malignant gliomas

\footnotetext{
* Correspondence: pfu@hust.edu.cn

${ }^{+}$Qing Zhang and Wei Xiang contributed equally to this work.

'Department of Neurosurgery, Union Hospital, Tongji Medical College,

Huazhong University of Science and Technology, Ave. Jiefang No.1277,

Wuhan 430022, People's Republic of China

Full list of author information is available at the end of the article
}

[5]. Radiotherapy has been performed to treat patients with intra-cranial tumours since the 1950s [6], but the technology has been slow to develop, and one of the hallmarks of this disease is radiotherapy-resistant glioma stem cells (GSCs) [4]. Temozolomide is the most common and effective chemotherapy agent for patients with malignant gliomas, but the results depend on the promoter methylation status of O6-methylguanine methyltransferase (MGMT) [5]. However, the complex features of malignant glioma contribute to a poor prognosis. Glioblastoma (GBM), which has the worst prognosis, only has a 14.6-month median survival rate [7]. In recent years, with the increased understanding of the biological features of gliomas, an increasing number of precise targeted therapies have been generated.

Different types of stem and progenitor cells, including neural stem cells (NSCs), mesenchymal stem cells (MSCs), haematopoietic progenitor cells (HPCs), and embryonic stem cells (ESCs), have been shown to have 
the capacity for tumour-tropic homing and migration both in vitro and in vivo [8-11]. In tumour microenvironments, tumour cells and stromal cells can secrete several factors, including transforming growth factor (TGF)- $\beta$, stromal cell-derived factor- $1 \alpha$ (SDF- $1 \alpha$ ), and vascular endothelial growth factor (VEGF), to recruit other cells into the tumour burden during tumour progression. Many reports have indicated that multipotent MSCs could migrate into tumour microenvironments in gliomas [12-14]. Based on the glioma tropism capacity, many basic and preclinical studies have used MSCs as cell vectors to deliver immune factors, anti-tumour proteins, anti-tumour microRNAs (miRNAs) or long non-coding RNAs (lncRNAs), suicide genes, and oncolytic viruses.

In this current review, we summarise the biological characteristics of human MSCs and present the principle of MSC-based therapy. Then, we analyse the current status and potential challenges in basic and preclinical studies for MSC-based therapy in patients with gliomas.

\section{Biological features of MSCs}

Friendenstein et al. reported the existence of MSCs in bone marrow in 1966 [15]. Subsequently, MSCs were widely and gradually found in many tissues [16-19]. MSCs were then gradually recognised as adult multipotent progenitor cells with the potential for self-renewal and differentiation into mesodermal and non-mesodermal lineages [20-22], but there was no definition marker for MSCs. In 2006, the International Society for Cellular Therapy (ISCT) defined the minimal criteria for MSCs according to their biological features: plastic adherent growth, positive expression of CD105, CD73, and CD90, negative expression of CD14, CD34, and CD45, and differentiation towards osteoblasts, adipocytes, and chondrocytes in vitro [23]. MSCs showed similar plastic adherent morphology from different sources [24], but they expressed slightly different cell surface markers, such as desmin, vascular endothelial (VE)-cadherin, $\alpha$-smooth muscle actin ( $\alpha$-SMA), nestin, and nerval/glial antigen (NG2) (Table 1) [25]. MSCs from different sources could differentiate into multiple cell lines, such as bone [26], muscle [27], adipose [28], tendons [29], neurons [30], and myocardium [31], under specific in-vivo and in-vitro conditions. The differentiation pathway to a particular phenotype can be regulated by some gene events (Fig. 1).

The regulation of the biological features of MSCs is complex and multi-layered. The microenvironment conditions in different sources is thought to be the main reason for the slight differences in biological characteristics and genome patterns of MSCs. In vitro, TGF- $\beta$, bone morphogenetic proteins, insulin, dexamethasone, and other differentiation factors could induce MSCs into multiple cell lines [32-35]. Several signalling pathways, such as the TGF- $\beta$ superfamily [36, 37], Hedgehog [38], and Wnt signalling [39], regulate and control the biological characteristics of MSCs. Recently, some new mechanisms, including miRNAs [40, 41] and lncRNAs [42], were reported to regulate the biological features. With a better understanding of MSCs, studies have aimed to modulate the biological characteristics of MSCs to treat several diseases, such as myocardial infarction, nerve injury, and arthritis.

\section{Tumour-specific tropism of MSCs}

Recently, tumour-associated MSCs (TA-MSCs) have been regarded as integral components involved in several hallmarks of different tumours, such as initiation, promotion, progression, and metastasis. Some of these TA-MSCs already exist in many tissues, but some of them are recruited into tumour microenvironments to take part in the progression of tumours via the production of various growth factors, chemokines, and cytokines and to cross-talk with tumour cells [43, 44].

MSCs are rarely found in the brains of normal mice and humans, and these MSCs with classical features are located in a vascular niche [25, 43]. An increasing number of studies have reported that MSCs could be recruited into the tumour microenvironment of gliomas. For example, SP-DiI-labelled human MSCs, but not fibroblasts, were detected in U87 tumour masses after intra-vascular or intra-cranial administration in vivo [45]. However, the exact mechanism of the tumour-tropism of MSCs in a glioma microenvironment has not yet been fully elucidated. SDF-1 $\alpha$, VEGF, platelet-derived growth factor (PDGF), endothelial cell growth factor (EGF),

Table 1 Cell surface markers of mesenchymal stem cells (MSCs) from four different sources

\begin{tabular}{lll}
\hline MSC source & Cell surface marker & References \\
\hline Bone marrow & Positive: SH2, SH3, CD29, CD44, CD71, CD73, CD124, CD90, CD105, CD106, CD120a & {$[12,19,24]$} \\
& Negative: CD34, CD45, CD19, CD3, HLA-DR, CD31, CD11b & {$[24,44,56,72]$} \\
Adipose tissue & Positive: CD13, CD29, CD44, CD73, HLA-I, CD90, CD105, CD166, HLA-ABC, & \\
& Negative: CD10, CD14, CD24, CD31, HLA-DR, CD36, CD38, CD45, CD49d, CD117, CD133, CD34, CD106, HLAll, SSEA4 & \\
Umbilical cord & Positive: CK8, CK18, CK19, CD10, CD13, HLA-I, CD29, CD73, CD105, CD106, CD90, CD44, CD73 & {$[9,16,24]$} \\
& Negative: CD14, CD31, CD33, CD34, HLA-DR, CD45, CD38, CD79, CD133, WWF & {$[25,85-87]$} \\
Human glioma & Positive: CD90, CD105, CD73, CD44, CD151, a-SMA, desmin, VE-cadherin, NG2, STRO-1, HLA-I & \\
& Negative: CD31, CD34, CD133, CD45, HLA-DR, CD14, CD19, Nestin, SMM & \\
\hline
\end{tabular}




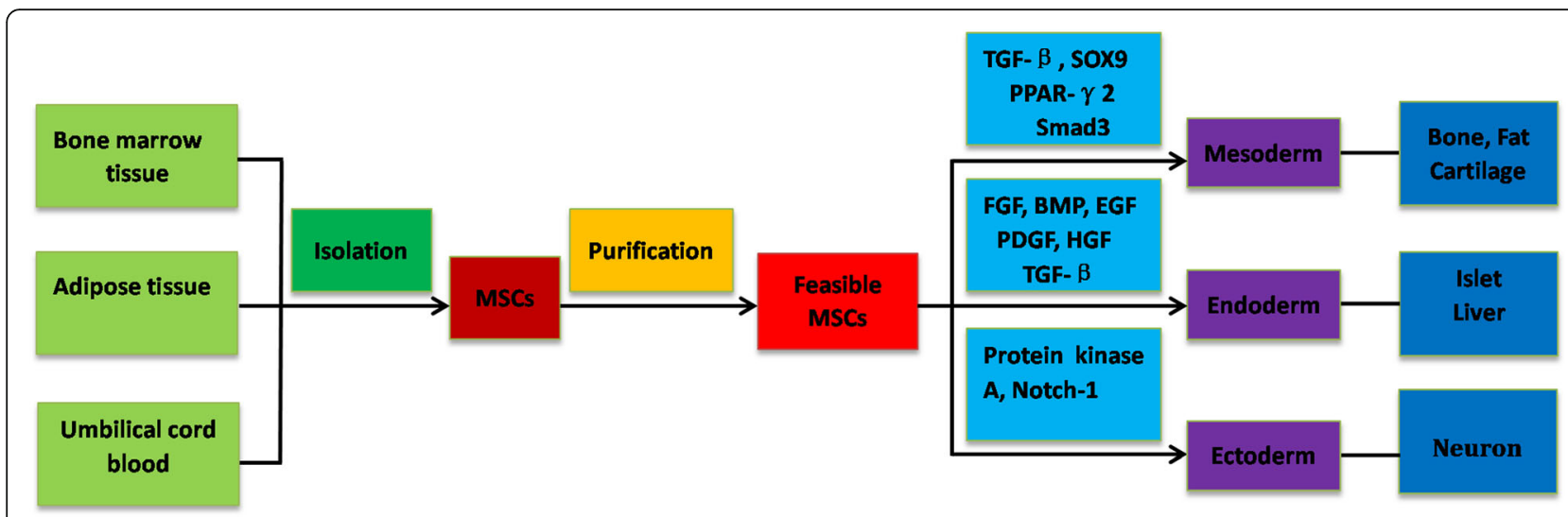

Fig. 1 Source of mesenchymal stem cells (MSCs) and various signalling pathways regulating MSC differentiation. Multiple signalling pathways and cytokines have also been found to be involved in lineage commitment. BMP bone morphogenetic protein, EGF endothelial growth factor, FGF fibroblast growth factor, HGF hepatocyte growth factor, PDGF platelet-derived growth factor, TGF transforming growth factor

TGF- $\beta 1$, interleukin (IL)-8, and monocyte chemoattractant protein-1 (MCP-1) have been found to be secreted by glioma cells or stromal cells and to contribute to tumour-tropism of MSCs [12-14].

Based on their tumour-tropism and facile ability to cross the blood-brain barrier, MSCs have been utilised as carrying vectors for glioma therapy.

\section{MSC-based therapy for gliomas}

MSC-based therapy for gliomas is a promising novel approach to transport different genes, proteins, and viruses. Various therapy approaches could suppress the growth of gliomas through different mechanisms, such as immunotherapy, suicide protein therapy, virus-based therapy, cytotoxic factor-based therapy, and anti-angiogenesis therapy, which are discussed more in the following sections.

\section{Immunotherapy}

Over the past decade, the ability of tumours to evade the immune system has always been a significant research direction for the rapid development of tumour immunotherapy. Malignant gliomas could escape the host immune system by secretion of immunosuppressive agents, inhibition of T-cell proliferation, and reduction in immune responses [46, 47]. Thus, the delivery of genes encoding cytokines, including interleukins (ILs) and interferon (IFN) family genes, to stimulate an immune response has been studied as an immunotherapy strategy in gliomas.

In 2004, Nakamura et al. found that intra-cranial administration of gene-modified MSCs expressing IL-2 could migrate towards a glioma site [48] and then infiltrate CD4 and CD8 lymphocytes, thus inducing strongly specific and curative anti-tumour immunity. Subsequently, researchers applied the same strategy using MSCs transduced to express other interleukins, such as IL-12, IL-7, and IL-18, and discovered that the anti-tumour effects of these agents were closely associated with enhancement of T-cell infiltration and tumour-specific T-cell responses, as well as the noting the occurrence of similar therapeutic efficacy in vivo [49-51]. In another attempt to target the immune system, Nakamizo et al. found that modified MSCs released soluble protein IFN- $\beta$, which significantly extended the survival of animals with established intra-cranial gliomas, and the inhibition of tumour cell growth correlated with dose-dependent increases [45].

This review provides a summary of currently open and recruiting MSC-based immune-therapy studies for gliomas, as shown in Table 2.The patterns of MSC-based therapy studies for glioma are particularly illuminated in Fig. 2. The results showed that MSCs can be genetically engineered to express cytokines and augment the immune response via enhancing $\mathrm{CD}^{+}$and $\mathrm{CD} 8^{+} \mathrm{T}$-cell infiltration and then stimulating subsequent cascade immune networks; these modified MSCs can be exploited to a therapeutic advantage against gliomas.

\section{Suicide protein-based therapy}

Suicide protein-based therapy is a widely applied form of gene therapy in the cancer field. This approach entails mRNA encoding a pro-drug-activating enzyme (suicide protein) transduced into MSCs, the injection of these MSCs into the tumour sites, and the subsequent conversion of non-toxic pro-drugs into toxic pro-drugs, leading to regression of tumour cells in vivo $[52,53]$. To date, the most commonly studied suicide genes in gliomas include herpes simplex virus thymidine kinase (HSV-TK) [54], cytosine deaminase/5-fluorocytosine (CD/5FC) [55], and rabbit carboxylesterase (rCE)/CPT-11 [56].

The HSV-TK/GCV system has been most reported in glioma treatment. This system is based on the ability of HSV-TK to efficiently phosphorylate the pro-drug ganciclovir to its monophosphate state, which is further 
Table 2 Summary of currently open and recruiting mesenchymal stem cell (MSC)-based immunotherapy studies for glioma

\begin{tabular}{|c|c|c|c|c|c|c|}
\hline MSC source (species) & Tumour type (species) & Route of administration & Experimental animal & Immunomodulatory gene & Year & References \\
\hline Bone marrow (rat) & $9 \mathrm{~L}$ (rat) & Intra-tumoural/contralateral & Fischer rat & $\mathrm{IL}-2$ & 2004 & {$[48]$} \\
\hline Bone marrow (rat) & N32 (rat) & Intra-tumoural & Fischer rat & IL-7 & 2010 & {$[50]$} \\
\hline Bone marrow (human) & U87 (human) & Intra-tumoural/intra-carotid & Nude mice & IFN- $\beta$ & 2005 & [45] \\
\hline Bone marrow (rat) & C6 (rat) & Intra-tumoural & Spraguee-Dawley rat & $\mathrm{IL}-18$ & 2009 & [49] \\
\hline $\begin{array}{l}\text { Umbilical cord blood } \\
\text { (human) }\end{array}$ & GL26 (mouse) & Contralateral/ipsilateral & C57BI/6 mice & $\mid \mathrm{L}-12$ & 2011 & {$[51]$} \\
\hline $\begin{array}{l}\text { Umbilical cord blood } \\
\text { (human) }\end{array}$ & U87 (human) & Intra-tumoural/contralateral & Nude mice & TRAIL & 2008 & [71] \\
\hline
\end{tabular}

IFN interferon, IL interleukin, TRAIL tumour necrosis factor-related apoptosis-inducing ligand

phosphorylated by cellular enzymes to GCV-triphosphate (GCV-TP) [57]. MSCs expressing HSV-TK would be more feasible for clinical applications than the method using NSC therapy [54].

Later, De Melo et al. designed a strategy using adipose-derived MSCs (AT-MSCs) expressing HSV-TK combined with GCV, which was able to exert a cytotoxic effect on U87 cells in vitro and diminish tumour size [58, 59]. Similarly, data has shown that a TK-MSC combination with valproic acid could selectively exert a profound bystander effect on glioblastoma cells in vivo and that it did not injure normal brain tissues $[60,61]$. This combined treatment significantly inhibited tumour growth and prolonged survival compared with glioma-bearing mice treated with MSC-TK in the absence of valproic acid (VPA) [58, 59].

Cytosine deaminase (CD) is another pro-drug-activating enzyme that can convert the non-toxic pro-drug 5-fluorocytosine (5-FC) to toxic 5-fluorouracil (5-FU), which effectively inhibits tumour growth. Early in 2012 a related study reported the use of CD-expressing MSCs combined with 5-FC for the treatment of intra-cranial rat gliomas and protected normal brain tissue from damage [62]. The CD/5-FC system demonstrated a potent bystander effect, with the ability to kill tumour cells even when the MSCs and tumour cells were not in direct contact, leading to the invading glioma cells becoming extensively disordered [63]. This system may represent a

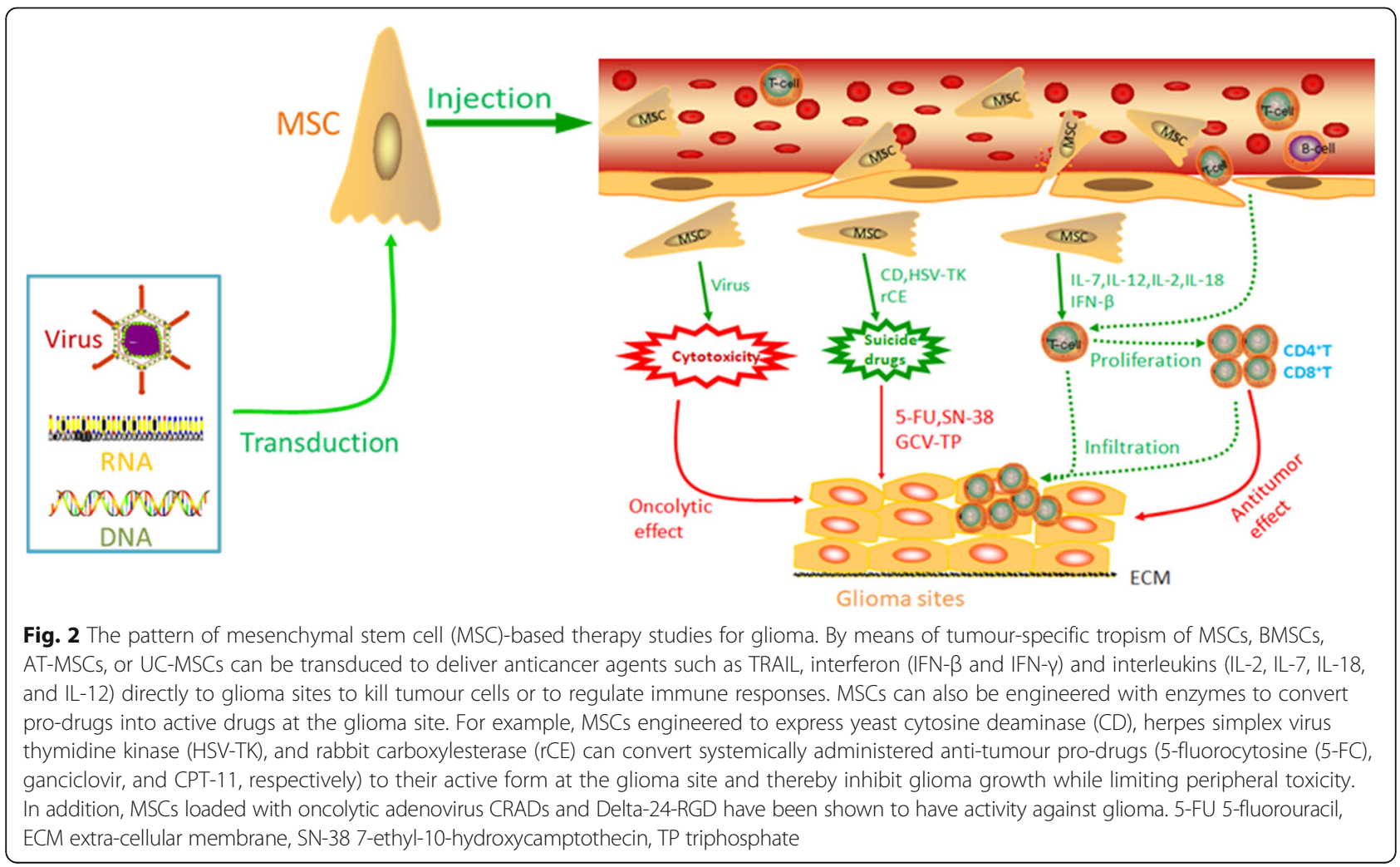


promising new therapeutic approach for highly invasive malignant gliomas. rCE enzymes can efficiently convert the pro-drug CPT-11 (irinotecan-7-ethyl-10-[4-(1-piperidino)-1-piperidino] carbonyloxycamptothecin) into the active drug SN-38 (7-ethyl-10-hydroxycamptothecin). Using the same enzyme/pro-drug therapy, Danks et al. explored intra-tumoural injection by combining genetically modified MSCs expressing rCE with CPT-11. The results showed that the therapy more effectively prolonged the survival of brain stem glioma-bearing rats than did treatment using only CPT-11 [64]. These strategies should provide an enhanced therapeutic effect for malignant gliomas.

\section{Virus-based therapy}

Oncolytic virotherapy is also a novel approach in which viruses are genetically modified to selectively replicate in tumour cells. The virus is released from its carrier at tumour sites and then selectively kills tumour cells and protects normal tissues from injury. However, the viral particles could be attacked and eliminated by the host immune system. In addition, the low-efficiency virus is far away from the tumour site. To overcome the barriers, tumour-tropic migratory cells may be used to deliver viral particles to the distant sites of tumours and shield a therapeutic virus from the host immune system [65].

Many studies using MSCs loaded with oncolytic adenovirus CRADs and Delta-24-RGD have demonstrated extended delivery of oncolytic viruses and prolonged survival of glioma-bearing animals treated with stem cell-mediated oncolytic virotherapy. In 2008, Sonabend et al. reported that MSCs provide CRAD delivery to distant glioma cells and that this delivery significantly enhanced the infection and apoptosis of tumour cells compared with injection of distant CRADs alone, showing a therapeutic advantage. Later, MSCs carrying $\Delta 24-R G D$ (hMSC- $\Delta 24$ ) were injected into the carotid artery of mice and then migrated to tumour sites, resulting in inhibited growth and improved survival of mice. Taken together, previous results indicated that MSCs migrate and deliver CRADs and Delta-24-RGD to distant glioma cells and improved the outcome of oncolytic virotherapy for glioma [66-69]. These results have consistently shown that virus-loaded MSCs are capable of migrating towards glioma sites and releasing viral particles that selectively infect tumour cells, the effects of which ultimately kill tumour cells.

In the future, there is a possibility that tumour-specific antigens will replace the use of viruses in which the anti-viral immune response is caused by carrier proteins, and thus the virus could combine the benefits of virotherapy with immune-therapy to achieve the aim of treating cancer.

\section{Cytotoxic factor-based therapy}

One novel strategy of tumour treatment is to induce apoptosis of tumour cells. Tumour necrosis factor (TNF)-related apoptosis-inducing ligand (TRAIL) is a member of the tumour necrosis factor (TNF) superfamily and could induce apoptosis of tumour cells through activation of the TNF/CD95L axis and spare the majority of non-malignant cells [70]. Studies have shown that MSCs expressing TRAIL could migrate towards a glioma, maintain their stem-like properties, induce the cytotoxic effects of glioma cells, and show prolonged survival in glioma animal models. TRAIL-secreting MSCs have the migration capacity towards tumour cells and can directly target glioma. In 2008, Kim et al. used umbilical cord-derived MSCs (UC-MSCs) expressing TRAIL to demonstrate a reduction in tumour volume and improvement in survival rate in glioma-bearing mice in vivo compared with controls [71]. Subsequently, Choi et al. reported the therapeutic efficacy and safety of TRAIL-producing human AT-MSCs against glioma, providing significant data for clinical trials using MSCs with therapeutic genes against brain gliomas [72]. The above studies demonstrate that MSCs expressing cytotoxic factors could migrate to tumour sites and induce apoptosis of tumour cells. Therefore, these results suggest that MSCs expressing TRAIL could provide an interesting approach for anti-glioma therapy.

\section{Anti-angiogenesis therapy}

Angiogenesis is an important characteristic event in the development and progression of gliomas. The use of factors that inhibit angiogenesis is thought to be one of the strategies for glioma treatment.

Pigment epithelial-derived factor (PEDF) is a $50-\mathrm{kDa}$ secreted glycoprotein that can activate the Fas/FasL pathway to induce endothelial cell death and regulate the balance between inducers and inhibitors of angiogenesis [73, 74]. Previously, Zhang et al. discovered that PEDF played an important role in angiogenesis and tumourigenesis of gliomas [75]. In 2013, Wang et al. proved that MSCs expressing PEDF effectively induced tumour cell apoptosis and inhibited angiogenesis, thereby decreasing tumour volume and prolonging the survival of glioma-bearing mice [74]. However, the molecular mechanism by which PEDF causes glioma apoptosis and anti-angiogenesis was not fully understood. Afterwards, Guo et al. found that the conditioned medium from phosphatase and tensin homologue (PTEN) mRNA-engineered MSCs induced U251 cell death via PI3K-AKT-mTOR pathways in vitro [76].

Thus, engineered MSCs act as an inhibitory molecular vehicle to promote apoptosis and attenuate angiogenesis in gliomas, and they may have the potential as a therapeutic agent in the clinical application of stem cell therapy against gliomas. 


\section{Potential challenges}

Although MSC-based therapy for malignant gliomas has become increasingly popular, all MSC-based therapies, similar to other stem cell-based therapies, appear to be limited in their effectiveness. Some recent studies on cross-talk between MSCs and glioma cells reveal the potential challenges to MSC-based therapy for gliomas.

\section{Do MSCs support or suppress glioma growth?}

There is no clear conclusion on whether MSCs themselves support or suppress the progression of glioma. AT-MSCs can increase the size of glioma tissue by a reduction in apoptosis and secretion of VEGF [77]. UC-MSCs can inhibit migration by the upregulation of PTEN and induce apoptosis of glioma cells by the downregulation of X-linked inhibitor of apoptosis (XIAP) [78]. Bone marrow-derived MSCs (BMSCs) can enhance the invasion of U373 but not U87 by overexpression of proteases [79]. AT-MSCs can promote the epithelial-mesenchymal transition in glioma cells [80]. Moreover, Schichor et al. found that the fusion of U87 and BMSCs facilitated the proliferation and migration of both in vitro, and the cross-talk of tumour cells and MSCs maintained the structural formation of syncytium [81]. Subsequently, Sun et al. showed that the fused cells gave rise to an enhanced angiogenesis of gliomas in vitro and in vivo and a stabilised vascular framework, implying an improvement in glioma growth [82].

Therefore, MSCs from different sources showed different abilities to promote or suppress the growth of glioma cells under different conditions. If these MSCs were investigated as delivery vectors, the effects of MSCs supporting or suppressing the growth of gliomas should also be considered.

\section{Glioma-associated MSCs (gbMSCs)}

gbMSCs were first isolated from fresh glioma tissues and identified by our team in 2014. We found that gbMSCs showed classical features of morphology, surface markers, and differentiation. The gbMSCs secrete different factors dependent on the inter-cellular cross-talk and hypoxia conditions [83]. Moreover, the percentage of gbMSCs in tumour samples correlated with the outcomes of patients with high-grade gliomas, whereas patients with a high percentage of gbMSCs had a poor overall survival rate [84]. Svensson et al. reported there were two distinct subpopulations of gbMSCs with different CD90 expression. CD90gbMSCs produced more VEGF and prostaglandin E2 (PGE2) than did $\mathrm{CD} 90^{+}$gbMSCs, and these two subpopulations had 211 differentially expressed genes but showed no mRNA differences [85]. Our team also found that CD90- gbMSCs could differentiate into pericytes and contribute to neovascularization in the glioma microenvironment [25]. CD90 ${ }^{+}$gbMSCs could increase the growth and invasion of glioma cells under a serum deprivation condition (data not shown). Figueroa et al. also found that
gbMSCs could increase the tumourigenicity of glioma stem cells by microRNA-1587 exosome transfer [86] and maintain the stemness of glioma stem cells by secretion of IL-6 [87]. All these data suggest the promoting role of gbMSCs in the aggression and progression of gliomas.

\section{Malignant transformation of MSCs}

As well as an assistant role of MSCs in tumour microenvironments, some studies have reported that MSCs can directly transform to malignant cells and have revealed a new challenge to MSC-based therapy for cancer. In 2004, Houghton et al. suggested that BMSCs were recruited to the sites of pre-cancerous lesions by inflammatory cytokines and then transformed into gastric cancer cells to promote cancer progression through a series of transformations [88]. Serakinci et al. reported that human adult MSCs had the neoplastic potential to contribute to mesenchymal tumour development after transducing the human telomerase reverse transcriptase (TERT) gene [89]. Recently, Tan et al. demonstrated that the proliferation and migration rate of the transformed mesenchymal stem cells (TMCs) were significantly increased compared with that of the MSCs in vitro and that TMCs led to tumourigenesis in an animal model [90]. These studies strongly suggest that MSC malignancy is possible under specific conditions, and there is a clear conclusion about the phenomenon and mechanism of malignant transformation of MSCs.

\section{MSC-mediated immunosuppression}

In tumour microenvironments, MSCs could support tumour growth by suppression of host immune responses. Once MSCs are recruited into a tumour microenvironment, they can secrete several ligands to help tumour cells build the immunosuppressive environment by recruiting monocytes, macrophages, and myeloid-derived suppressor cells. These immune cells then inhibit the anti-tumour immune responses of $\mathrm{T}$ cells [43]. In gliomas, some researchers found that $\mathrm{CD}^{-} 0^{-}$gbMSCs showed stronger ability for tumour immunosuppression than their $\mathrm{CD}^{+} 0^{+}$ counterparts [85]. However, there are still too few publications about the suppressive role of MSCs in glioma.

\section{Conclusions}

Currently, MSC-based therapy for gliomas has become an increasing focus of research. MSCs are regarded as effective vectors to deliver therapeutic agents to tumour sites. Despite MSC-related studies making great strides in the field of glioma treatment, there remain some powerful challenges. Moreover, there are no on-going clinical trials on MSC-based therapy for malignant gliomas. In conclusion, MSC-based therapy for glioma is still in its infancy, and we need a better understanding of the biological consequences of MSC-based therapy before it is widely used in the treatment of patients with malignant gliomas. 


\section{Abbreviations}

5-FC: 5-Fluorocytosine; AT-MSC: Adipose-derived mesenchymal stem cell; BMSC: Bone marrow-derived mesenchymal stem cell; CD: Cytosine deaminase; EGF: Endothelial cell growth factor; ESC: Embryonic stem cell; gbMSC: Glioma-associated mesenchymal stem cell; GSC: Glioma stem cell; HPC: Haematopoietic progenitor cell; HSV-TK: Herpes simplex virus thymidine kinase; IFN: Interferon; IL: Interleukin; MCP-1: Monocyte chemoattractant protein-1; MSC: Mesenchymal stem cell; NSC: Neural stem cell; PDGF: Plateletderived growth factor; PEDF: Pigment epithelial-derived factor; PGE2: Prostaglandin E2; PTEN: Phosphatase and tensin homologue; rCE: Rabbit carboxylesterase; SDF-1a: Stromal cell-derived factor-1a; TA-MSC: Tumour-associated mesenchymal stem cell; TGF: Transforming growth factor; TMC: Transformed mesenchymal stem cell; TNF: Tumour necrosis factor; TRAIL: Tumour necrosis factor-related apoptosis-inducing ligand; UC-MSC: Umbilical cord-derived mesenchymal stem cell; VEGF: Vascular endothelial growth factor; XIAP: X-linked inhibitor of apoptosis

\section{Funding}

This work was partly supported by a grant from the National Natural Science Foundation of China (no. 81572488) to WX and grants from the Science and Technology Department of Hubei Province (2015CFB458 and 2017CFB268) to PF.

\section{Authors' contributions}

QZ and WX are responsible for the conception, draft, and modification of the manuscript; DYY, BZX, WWW, AA, NXX, XBJ, and HYZ equally contributed to the collection and sorting of the data and documents; PF manipulated the polishing of the language and writing style and managed the integral vision and top-layer design of the manuscript. All authors read and approved the final manuscript

\section{Ethics approval and consent to participate}

Not applicable.

\section{Consent for publication}

All authors agreed to publication.

\section{Competing interests}

The authors declare that they have no competing interests.

\section{Publisher's Note}

Springer Nature remains neutral with regard to jurisdictional claims in published maps and institutional affiliations.

\section{Author details}

'Department of Neurosurgery, Union Hospital, Tongji Medical College, Huazhong University of Science and Technology, Ave. Jiefang No.1277, Wuhan 430022, People's Republic of China. ${ }^{2}$ Department of Cardiology, Beijing Anzhen Hospital, Capital Medical University, No. 2, Anzhen Road, Chaoyang District, Beijing 100029, People's Republic of China.

\section{Published online: 24 August 2018}

\section{References}

1. Barbarin A, Seite P, Godet J, et al. Atypical nuclear localization of VIP receptors in glioma cell lines and patients. Biochem Biophys Res Commun. 2014;454: 524-30.

2. Schwartzbaum JA, Fisher JL, Aldape KD, et al. Epidemiology and molecular pathology of glioma. Nat Clin Pract Neurol. 2006:2:494-503.

3. Kassebaum NJ, Bernabe E, Dahiya M, et al. Global burden of severe periodontitis in 1990-2010: a systematic review and meta-regression. J Dent Res. 2014;93:1045-53.

4. Anjum K, Shagufta BI, Abbas SQ, et al. Current status and future therapeutic perspectives of glioblastoma multiforme (GBM) therapy: a review. Biomed Pharmacother. 2017;92:681-9.

5. Wick W, Platten M, Meisner C, et al. Temozolomide chemotherapy alone versus radiotherapy alone for malignant astrocytoma in the elderly: the NOA-08 randomised, phase 3 trial. Lancet Oncol. 2012;13:707-15.

6. Pouyanne L, Pouyanne $H$, Marchand. Malignant glioma of the left cerebral hemisphere in an 18-month-old infant; removal, radiotherapy, improvement J Med Bord. 1950;127:261-3.
7. Surawicz TS, Davis F, Freels S, et al. Brain tumor survival: results from the National Cancer Data Base. J Neuro-Oncol. 1998:40:151-60.

8. Aboody KS, Brown A, Rainov NG, et al. Neural stem cells display extensive tropism for pathology in adult brain: evidence from intracranial gliomas. Proc Natl Acad Sci U S A. 2000;97:12846-51.

9. Fan C, Wang D, Zhang Q, et al. Migration capacity of human umbilical cord mesenchymal stem cells towards glioma in vivo. Neural Regen Res. 2013;8: 2093-102.

10. Serfozo P, Schlarman MS, Pierret C, et al. Selective migration of neuralized embryonic stem cells to stem cell factor and media conditioned by glioma cell lines. Cancer Cell Int. 2006:6:1.

11. Tabatabai G, Hasenbach K, Herrmann C, et al. Glioma tropism of lentivirally transduced hematopoietic progenitor cells. Int J Oncol. 2010;36:1409-17.

12. Schichor C, Birnbaum T, Etminan N, et al. Vascular endothelial growth factor a contributes to glioma-induced migration of human marrow stromal cells (hMSC). Exp Neurol. 2006;199:301-10.

13. Kim DS, Kim JH, Lee JK, et al. Overexpression of CXC chemokine receptors is required for the superior glioma-tracking property of umbilical cord bloodderived mesenchymal stem cells. Stem Cells Dev. 2009:18:511-9.

14. Xu F, Shi J, Yu B, et al. Chemokines mediate mesenchymal stem cell migration toward gliomas in vitro. Oncol Rep. 2010;23:1561-7.

15. Friedenstein AJ, Piatetzky S II, Petrakova KV. Osteogenesis in transplants of bone marrow cells. J Embryol Exp Morphol. 1966;16:581-390.

16. Rogers I, Casper RF. Umbilical cord blood stem cells. Best Pract Res Clin Obstet Gynaecol. 2004;18:893-908.

17. Bussolati B, Bruno S, Grange C, et al. Isolation of renal progenitor cells from adult human kidney. Am J Pathol. 2005;166:545-55.

18. Wang XJ, Xiang BY, Ding YH, et al. Human menstrual blood-derived mesenchymal stem cells as a cellular vehicle for malignant glioma gene therapy. Oncotarget. 2017;8:58309-21.

19. Wexler SA, Donaldson C, Denning-Kendall $P$, et al. Adult bone marrow is a rich source of human mesenchymal 'stem' cells but umbilical cord and mobilized adult blood are not. Br J Haematol. 2003;121:368-74.

20. Chen $L B$, Jiang $X B$, Yang $L$. Differentiation of rat marrow mesenchymal stem cells into pancreatic islet beta-cells. World J Gastroenterol. 2004;10:3016-20.

21. Lee KD, Kuo TK, Whang-Peng J, et al. In vitro hepatic differentiation of human mesenchymal stem cells. Hepatology. 2004;40:1275-84.

22. Pittenger MF, Mackay AM, Beck SC, et al. Multilineage potential of adult human mesenchymal stem cells. Science. 1999;284:143-7.

23. Dominici M, Le Blanc K, Mueller I, et al. Minimal criteria for defining multipotent mesenchymal stromal cells. The International Society for Cellular Therapy position statement. Cytotherapy. 2006;12:315-7.

24. Mushahary D, Spittler A, Kasper C, et al. Isolation, cultivation, and characterization of human mesenchymal stem cells. Cytometry A. 2018;93:19-31.

25. Yi D, Xiang W, Zhang Q, et al. Human glioblastoma-derived mesenchymal stem cell to pericytes transition and angiogenic capacity in glioblastoma microenvironment. Cell Physiol Biochem. 2018;46:279-90.

26. Bruder SP, Kurth AA, Shea M, et al. Bone regeneration by implantation of purified, culture-expanded human mesenchymal stem cells. J Orthop Res. 1998;16:155-62.

27. Ferrari G, Cusella-De Angelis $\mathrm{G}$, Coletta $\mathrm{M}$, et al. Muscle regeneration by bone marrow-derived myogenic progenitors. Science. 1998;279:1528-30.

28. Dennis JE, Merriam A, Awadallah A, et al. A quadripotential mesenchymal progenitor cell isolated from the marrow of an adult mouse. J Bone Miner Res Off J Am Soc Bone Miner Res. 1999:14:700-9.

29. Young RG, Butler DL, Weber W, et al. Use of mesenchymal stem cells in a collagen matrix for Achilles tendon repair. J Orthop Res. 1998;16:406-13.

30. Qian L, Saltzman WM. Improving the expansion and neuronal differentiation of mesenchymal stem cells through culture surface modification. Biomaterials. 2004;25:1331-7.

31. Miao C, Lei M, Hu W, et al. A brief review: the therapeutic potential of bone marrow mesenchymal stem cells in myocardial infarction. Stem Cell Res Ther. 2017;8:242-5

32. Fiorentini E, Granchi D, Leonardi E, et al. Effects of osteogenic differentiation inducers on in vitro expanded adult mesenchymal stromal cells. Int J Artif Organs. 2011;34:998-1011.

33. Pelttari K, Steck E, Richter W. The use of mesenchymal stem cells for chondrogenesis. Injury. 2008:39:58-65.

34. Contador D, Ezquer F, Espinosa M, et al. Dexamethasone and rosiglitazone are sufficient and necessary for producing functional adipocytes from mesenchymal stem cells. Exp Biol Med (Maywood). 2015;240:1235-46. 
35. Banerjee $\mathrm{C}$, Javed A, Choi JY, et al. Differential regulation of the two principal Runx2/Cbfa1 n-terminal isoforms in response to bone morphogenetic protein-2 during development of the osteoblast phenotype. Endocrinology. 2001;142:4026-39.

36. Valcourt $\mathrm{U}$, Moustakas A. BMP signaling in osteogenesis, bone remodeling and repair. Eur J Trauma. 2005;31:464-79.

37. Sampath TK, Maliakal JC, Hauschka PV, et al. Recombinant human osteogenic protein-1 (hOP-1) induces new bone formation in vivo with a specific activity comparable with natural bovine osteogenic protein and stimulates osteoblast proliferation and differentiation in vitro. J Biol Chem. 1992;267:20352-62.

38. St-Jacques B, Hammerschmidt M, McMahon AP. Indian hedgehog signaling regulates proliferation and differentiation of chondrocytes and is essential for bone formation. Genes Dev. 1999;13:2072-86.

39. Ling L, Nurcombe V, Cool SM. Wnt signaling controls the fate of mesenchymal stem cells. Gene. 2009;433:7.

40. Benfey PN. Molecular biology: microRNA is here to stay. Nature. 2003;425: 244-5.

41. Li Z, Hassan MQ, Volinia S, et al. A microRNA signature for a BMP2-induced osteoblast lineage commitment program. Proc Natl Acad Sci U S A. 2008; 105:13906-11.

42. Antoniou D, Stergiopoulos A, Politis PK. Recent advances in the involvement of long non-coding RNAs in neural stem cell biology and brain pathophysiology. Front Physiol. 2014;5:8

43. Shi $Y, D u L$, Lin $L$, et al. Tumour-associated mesenchymal stem/stromal cells: emerging therapeutic targets. Nat Rev Drug Discov. 2017;16:35-52.

44. Langroudi L, Hassan ZM, Soleimani M, et al. Tumor associated mesenchymal stromal cells show higher immunosuppressive and angiogenic properties compared to adipose derived MSCs. Iran J Immunol. 2015;12:226-39.

45. Nakamizo A, Marini F, Amano T, et al. Human bone marrow-derived mesenchymal stem cells in the treatment of gliomas. Cancer Res. 2005;65: 3307-18.

46. Razavi SM, Lee KE, Jin BE, et al. Immune evasion strategies of glioblastoma. Front Surg. 2016:3:9.

47. Codrici E, Enciu AM, Popescu ID, et al. Glioma stem cells and their microenvironments: providers of challenging therapeutic targets. Stem Cells Int. 2016;2016:20.

48. Nakamura K, Ito Y, Kawano Y, et al. Antitumor effect of genetically engineered mesenchymal stem cells in a rat glioma model. Gene Ther. 2004;11:1155-64

49. $X u$ G, Jiang $X D, X u Y$, et al. Adenoviral-mediated interleukin-18 expression in mesenchymal stem cells effectively suppresses the growth of glioma in rats. Cell Biol Int. 2009;33:466-74.

50. Gunnarsson S, Bexell D, Svensson A, et al. Intratumoral IL-7 delivery by mesenchymal stromal cells potentiates IFNgamma-transduced tumor cell immunotherapy of experimental glioma. J Neuroimmunol. 2010;218:140-4.

51. Ryu CH, Park SH, Park SA, et al. Gene therapy of intracranial glioma using interleukin 12-secreting human umbilical cord blood-derived mesenchymal stem cells. Hum Gene Ther. 2011:22:733-43.

52. Hall J, Prabhakar S, Balaj L, et al. Delivery of therapeutic proteins via extracellular vesicles: review and potential treatments for Parkinson's disease, glioma, and schwannoma. Cell Mol Neurobiol. 2016;36:417-27.

53. Dixit K, Kumthekar P. Gene delivery in Neuro-oncology. Curr Oncol Rep. 2017;19:11.

54. Amano S, Li S, Gu C, et al. Use of genetically engineered bone marrowderived mesenchymal stem cells for glioma gene therapy. Int J Oncol. 2009; 35:1265-70.

55. Fei S, Qi X, Kedong S, et al. The antitumor effect of mesenchymal stem cells transduced with a lentiviral vector expressing cytosine deaminase in a rat glioma model. J Cancer Res Clin Oncol. 2012;138:347-57.

56. Choi SA, Lee JY, Wang KC, et al. Human adipose tissue-derived mesenchymal stem cells: characteristics and therapeutic potential as cellular vehicles for prodrug gene therapy against brainstem gliomas. Eur J Cancer. 2012;48:129-37.

57. Namba H, Kawaji H, Yamasaki T. Use of genetically engineered stem cells for glioma therapy. Oncol Lett. 2016;11:9-15.

58. Bovenberg MS, Degeling MH, Tannous BA. Advances in stem cell therapy against gliomas. Trends Mol Med. 2013;19:281-91.

59. De Melo SM, Bittencourt S, Ferrazoli EG, et al. The anti-tumor effects of adipose tissue mesenchymal stem cell transduced with HSV-Tk gene on U-87-driven brain tumor. PLoS One. 2015;10:13.
60. Matuskova M, Hlubinova K, Pastorakova A, et al. HSV-tk expressing mesenchymal stem cells exert bystander effect on human glioblastoma cells. Cancer Lett. 2010;290:58-67.

61. Uchibori R, Okada T, Ito T, et al. Retroviral vector-producing mesenchymal stem cells for targeted suicide cancer gene therapy. J Gene Med. 2009;11: 373-81.

62. Kosaka H, Ichikawa T, Kurozumi K, et al. Therapeutic effect of suicide genetransferred mesenchymal stem cells in a rat model of glioma. Cancer Gene Ther. 2012;19:572-8.

63. Jung JH, Kim AA, Chang DY, et al. Three-dimensional assessment of bystander effects of mesenchymal stem cells carrying a cytosine deaminase gene on glioma cells. Am J Cancer Res. 2015;5:2686-96.

64. Danks MK, Yoon KJ, Bush RA, et al. Tumor-targeted enzyme/prodrug therapy mediates long-term disease-free survival of mice bearing disseminated neuroblastoma. Cancer Res. 2007:67:22-5.

65. Yamamoto M, Curiel DT. Current issues and future directions of oncolytic adenoviruses. Mol Ther. 2010;18:243-50.

66. Sonabend AM, Ulasov IV, Tyler MA, et al. Mesenchymal stem cells effectively deliver an oncolytic adenovirus to intracranial glioma. Stem Cells. 2008;26: 831-41.

67. Parker Kerrigan BC, Shimizu Y, Andreeff M, et al. Mesenchymal stromal cells for the delivery of oncolytic viruses in gliomas. Cytotherapy. 2017;19:445-57.

68. Yong RL, Shinojima N, Fueyo J, et al. Human bone marrow-derived mesenchymal stem cells for intravascular delivery of oncolytic adenovirus Delta24-RGD to human gliomas. Cancer Res. 2009;69:8932-40.

69. Jiang H, Gomez-Manzano C, Lang FF, et al. Oncolytic adenovirus: preclinical and clinical studies in patients with human malignant gliomas. Curr Gene Ther. 2009:9:422-7.

70. Tang XJ, Lu JT, Tu HJ, et al. TRAIL-engineered bone marrow-derived mesenchymal stem cells: TRAIL expression and cytotoxic effects on C6 glioma cells. Anticancer Res. 2014;34:729-34

71. Kim SM, Lim JY, Park SI, et al. Gene therapy using TRAlL-secreting human umbilical cord blood-derived mesenchymal stem cells against intracranial glioma. Cancer Res. 2008:68:9614-23.

72. Choi SA, Lee YE, Kwak PA, et al. Clinically applicable human adipose tissuederived mesenchymal stem cells delivering therapeutic genes to brainstem gliomas. Cancer Gene Ther. 2015;22:302-11.

73. Ren JG, Jie C, Talbot C. How PEDF prevents angiogenesis: a hypothesized pathway. Med Hypotheses. 2005;64:74-8.

74. Wang Q, Zhang Z, Ding T, et al. Mesenchymal stem cells overexpressing PEDF decrease the angiogenesis of gliomas. Biosci Rep. 2013;33:199-205.

75. Zhang T, Guan M, Lu Y. Production of active pigment epithelium-derived factor in E. Coli. Biotechnol Lett. 2005;27:403-7.

76. Guo XR, Hu QY, Yuan YH, et al. PTEN-mRNA engineered mesenchymal stem cell-mediated cytotoxic effects on U251 glioma cells. Oncol Lett. 2016;11:2733-40.

77. Ridge SM, Sullivan FJ, Glynn SA. Mesenchymal stem cells: key players in cancer progression. Mol Cancer. 2017;16:31.

78. Dasari VR, Velpula KK, Kaur K, et al. Cord blood stem cell-mediated induction of apoptosis in glioma downregulates X-linked inhibitor of apoptosis protein (XIAP). PLoS One. 2010;5:e11813.

79. Breznik B, Motaln H, Vittori M, et al. Mesenchymal stem cells differentially affect the invasion of distinct glioblastoma cell lines. Oncotarget. 2017;8: 25482-99.

80. Iser IC, Ceschini SM, Onzi GR, et al. Conditioned medium from adiposederived stem cells (ADSCS) promotes epithelial-to-mesenchymal-like transition (EMT-like) in glioma cells in vitro. Mol Neurobiol. 2016;53:7184-99.

81. Schichor C, Albrecht $V$, Korte B, et al. Mesenchymal stem cells and glioma cells form a structural as well as a functional syncytium in vitro. Exp Neurol. 2012;234:208-19.

82. Sun C, Zhao D, Dai $X$, et al. Fusion of cancer stem cells and mesenchymal stem cells contributes to glioma neovascularization. Oncol Rep. 2015;34: 2022-30.

83. Guo KT, Fu P, Juerchott K, et al. The expression of Wnt-inhibitor DKK1 (Dickkopf 1 ) is determined by intercellular crosstalk and hypoxia in human malignant gliomas. J Cancer Res Clin Oncol. 2014;140:1261-70.

84. Shahar T, Rozovski U, Hess KR, et al. Percentage of mesenchymal stem cells in high-grade glioma tumor samples correlates with patient survival. NeuroOncology. 2017;19:660-8.

85. Svensson A, Ramos-Moreno T, Eberstal S, et al. Identification of two distinct mesenchymal stromal cell populations in human malignant glioma. J Neuro-Oncol. 2017;131:245-54. 
86. Figueroa J, Phillips LM, Shahar T, et al. Exosomes from glioma-associated mesenchymal stem cells increase the tumorigenicity of glioma stem-like cells via transfer of miR-1587. Cancer Res. 2017;77:5808-19.

87. Hossain A, Gumin J, Gao F, et al. Mesenchymal stem cells isolated from human gliomas increase proliferation and maintain stemness of glioma stem cells through the IL-6/gp130/STAT3 pathway. Stem Cells. 2015;33: 2400-15.

88. Houghton JM, Stoicov C, Nomura S, et al. Gastric cancer originating from bone marrow-derived cell. Science. 2004;306:1568-71.

89. Serakinci N, Guldberg P, Burns JS, et al. Adult human mesenchymal stem cell as a target for neoplastic transformation. Oncogene. 2004;23:5095-8.

90. Tan B, Shen L, Yang K, et al. C6 glioma-conditioned medium induces malignant transformation of mesenchymal stem cells: possible role of S100B/RAGE pathway. Biochem Biophys Res Commun. 2018:495:78-85. 